\title{
Observational white dwarf seismology
}

\author{
S. O. Kepler \\ Instituto de Física, Universidade Federal do Rio Grande do Sul, Porto Alegre, RS - Brazil
}

\begin{abstract}
After the Sun, the stars with the most detected pulsation modes are the white dwarfs. From the hot PG 1159 stars, through the pulsating DBs near $25000 \mathrm{~K}$, to the more numerous DAVs around $12000 \mathrm{~K}$, we now know of around 150 pulsating white dwarf stars, but they are still all in the nearby thin disk of our Galaxy. As the white dwarf models are simple and the details of the initial conditions are washed out when the stars reach the DBV and the DAV instability strips, seismology does give structural information with detail and precision, and even allows us to measure evolutionary timescales. Taking into account that around $97 \%$ of all stars evolve to white dwarfs, we measure the records of Galactic history, which is a powerful tool to study physics at high energies.
\end{abstract}

\section{Introduction}

Asteroseismology of white dwarf stars is a strong tool for probing high energy and high density physics, such as the study of neutrinos (weak interaction - Kawaler 1997; O'Brien et al. 1998; Winget et al. 2004), axions (the best candidate for cold dark matter, Córsico et al. 2001), crystallization (cool white dwarf stars are quantum crystals, Winget et al. 1997; Córsico et al. 2004, 2005; Kanaan et al. 2005) and even the determination of the $\mathrm{C}(\alpha, \gamma)$ cross section (Metcalfe, Salaris \& Winget 2002; Metcalfe 2003; Metcalfe, Montgomery \& Kawaler 2003) essential in the study of type Ia supernovae.

In terms of stellar structure and evolution, the observed pulsations can be used to evaluate the total mass, the layers and even core masses, rotation periods, magnetic fields, differential rotation (Kawaler, Sekii \& Gough 1999), and also a real measurement of the evolutionary time scales, $\mathrm{d} R / \mathrm{d} t$ (Costa et al. 2003) and $\mathrm{d} T / \mathrm{d} t$ (Stover et al. 1980; Kepler et al. 1982, 2005b; Mukadam et al. 2003), which in turn can be used to measure the age of the Galaxy (Winget et al. 1987; Hansen et al. 2002). The changes in pulsation periods of white dwarf stars can also be used for the detection of extra-solar planets, complementing the search space not easily available for radial velocity measurements (Winget et al. 2003).

The pre-white dwarf PG 1159 stars around $75000 \mathrm{~K}$ to $170000 \mathrm{~K}$ have the largest number of modes detected. With the first class of pulsating stars to be predicted theoretically before discovery, the DBVs around $22000 \mathrm{~K}$ to $29000 \mathrm{~K}$, and the first pulsating white dwarf stars to be discovered, serendipitously, back in 1968 , the DAVs around $10850 \mathrm{~K}$ to $12270 \mathrm{~K}$, the 150 pulsating white dwarf stars known are all in the thin disk of our Galaxy, just because they are intrinsically faint. They form the most numerous class of variable stars. As their structure is simple, seismology does give structural information with detail and precision. Because of their high densities and internal temperatures, they are tools to study physics at high energies, where quantum effects are dominant, but post-Newtonian corrections are still not dominant.

All the pulsating white dwarf stars are non-radial g-mode pulsators, and the eigenmodes are described by three indices: the number of radial nodes $(k)$, the total number of nodes across the surface $(\ell)$, and the number of azimuthal nodes $(\mathrm{m})$. With the mode identification via multiplets for pulsating PG 1159 stars and DBVs, or via chromatic amplitudes changes from ultraviolet to optical (Kepler et al. 2000; Castanheira et al. 2004, 2005), or line profile 
variations (Clemens, van Kerkwijk, \& Wu, 2000; Kotak et al. 2002, 2003; Kotak, van Kerkwijk \& Clemens 2002, 2004; Thompson et al. 2003), for DBVs and DAVs, we have been successful in applying seismology to estimate the mass, and total luminosity via the mass-radius relation, and consequently the distance, but also the thickness of the composition layers, including the core composition, and rotation periods. Nearly all the modes identified up to today have $\ell=1$ or 2 . Yeates et al. (2005) propose to use the amplitudes of the combination peaks to identify $\ell=1$, using the amplitude equations of Wu (2001).

Hydrogen-atmosphere white dwarf stars (DAs) comprise $\sim 90 \%$ of all white dwarf stars; helium dominated DOs and DBs total close to the remaining 10\% (Eisenstein et al. 2006).

\section{Pulsating PG 1159 stars}

The instability strip of the pulsating PG 1159 stars, or GW Vir stars (McGraw et al. 1979), around $T_{\text {eff }} \simeq 170000 \mathrm{~K}$ to $75000 \mathrm{~K}$ and $\log g=5.7$ to 7.5 , include both the DOVs (McGraw et al. 1979, Bond et al. 1984), without evidence of surrounding planetary nebulae, and the PNNVs (Grauer \& Bond 1984), both with detectable evidence of ongoing mass loss. Their atmospheres are mainly composed of $\mathrm{He}, \mathrm{C}$ and $\mathrm{O}$, and the pulsators also have strong lines of N (Dreizler 1998). These hydrogen deficient stars are probably the evolutionary remnants of a born again episode, triggered by a late helium thermal pulse after the star has left the AGB (Fujimoto 1977; Schönberner 1979; Iben 1982; and Althaus et al. 2005). There are 11 pulsators known, and their periods change slowly with time due to variations in both temperature, probably dominant, and radius

$$
\frac{\mathrm{d} P}{\mathrm{~d} t}=a \frac{\mathrm{d} T}{\mathrm{~d} r}+b \frac{\mathrm{d} R}{\mathrm{~d} t}
$$

(Winget, Hansen \& van Horn 1983; Winget et al. 1985; Kawaler et al. 1986; Costa et al. 1999). The pulsation periods range from 7 to 50 minutes, being longer for the PNNVs (Vauclair, Solheim \& Østensen 2005) and, for the prototype, have been detected even in X-ray (Barstow et al. 1986). The period spacings for this class of variables are mainly given by asymptotic theory, as they are high-k pulsators. Presently, the largest uncertainty in the mass determination from the period spacings is coming from uncertainty in the theoretical models (Kawaler et al. 1995, 2004), not due to observational precision. So an effort in accurate modelling is necessary and hopefully in progress. Note that the accuracy in the mass determination from the period spacings, even with the uncertainty in the models, of the order of $\Delta M \simeq 0.02 M_{\odot}$ (Costa et al. 2003), is at least an order of magnitude more accurate than the determinations from spectral fitting. As convection is negligible in these stars, the $\kappa-\gamma$ mechanism at the $\mathrm{C}$ and $\mathrm{O}$ partial ionization zones are the main drivers, as originally proposed by Starrfield et al. (1983) and confirmed by Bradley \& Dziembowski (1996), and more accurately with the evolutionary models of Quirion et al. $(2004,2005,2006,2007)$, Córsico \& Althaus $(2005,2006)$ and Córsico, Althaus \& Miller Bertolami (2006).

\section{DBVs}

The class of pulsating DB stars, also called V777 Her after their progenitor GD 358, discovered by Winget et al. (1982a), with an atmosphere of helium, has 13 pulsators known $(+4$ strong candidates - Nitta et al. 2005). The instability strip is located around $T_{\text {eff }} \simeq 29000 \mathrm{~K}$ to $22000 \mathrm{~K}$, with an uncertainty around $2000 \mathrm{~K}$ due to uncertainties in the temperature determination from spectral fitting (Beauchamp et al. 1999; Castanheira et al. 2006a). The excitation is due to the $\kappa-\gamma$ mechanism in the He partial ionization zone, as proposed by Winget et al. (1982b), and is the first class of variable stars predicted theoretically. The pulsation spectra, in general, show a large number of harmonics and combination periodicities, 
consistent with a thick convection zone distorting the eigenmodes that enter the base of the convection zone (Ising \& Koester 2001; Montgomery 2005, 2006).

The prototype and brightest known member, GD 358, shows hundreds of combination peaks in the Fourier transform of the light curve, and shows strong amplitude changes on timescales of weeks and months (Winget et al. 1994; Vuille et al. 2000; Kepler et al. 2003). The periods range from 140 to around $1000 \mathrm{~s}$ and the uncertainties in temperatures, coupled with the contamination of a small amount of hydrogen, if any, in the spectra of a few DBs, makes the analysis of the purity of the DB instability strip difficult.

\section{DAVs}

The DAVs or ZZ Ceti class of pulsating white dwarf stars, with 126 known members in November 2006 (Mukadam et al. 2004; Mullally et al. 2005; Kepler et al. 2005a; Gianninas et al. 2005; Voss et al. 2006; Castanheira et al. 2006bc), was the first observed, when Arlo Landolt (1968) was studying the photometric standard star HL Tau 76 and found variations of up to 0.3 mag on time scales around 12 minutes. Soon afterwards, Lasker \& Hesser (1969) found G44-32, with periods around 10 and 13.7 minutes, followed by R $548=$ ZZ Ceti, with periods of 213s and 271s (Lasker \& Hesser 1971). Warner \& Robinson (1972) and Chanmugam (1972) proposed the pulsations were non radial g-modes, as both the radial pulsations and p-modes should have much shorter periods in white dwarf stars. The class was first studied by McGraw \& Robinson (1976). Robinson, Nather \& McGraw (1976) first detected rotational splittings, in R 548, and McGraw (1979) and Robinson, Kepler \& Nather (1982) showed the light variations were dominated by changes in temperatures caused by g-mode pulsations. The filter mechanism that selects which modes get excited to observable amplitudes, mode trapping, was studied by Winget et al. (1981) and Córsico et al. (2002). Some pulsators have small amplitudes and sinusoidal light curves (Stover et al. 1980; Kepler et al. 1982, 1983; Kepler 1984), while others are high amplitude pulsators, with many harmonics and combination peaks detected (McGraw \& Robinson 1975; Robinson et al. 1978; Kleinman et al. 1998; Vuille 2000; Dolez et al. 2006).

The nonadiabatic models of Dziembowski (1977), Keeley (1979), Dziembowski \& Koester (1981), Dolez \& Vauclair (1981) and Winget et al. (1982b) concluded the excitation was due to the $\kappa-\gamma$ mechanism in the hydrogen partial ionization zone, but in recent calculations with OP and OPAL opacities, the models indicate that the convection zone is carrying about $90 \%$ of the flux even at the blue edge, and totally dominates the driving, i.e. convective driving in the convection zone caused the partial ionization zone, as proposed by Brickhill (1991) and Goldreich \& Wu (1999). The question of the purity of the ZZ Ceti instability strip also depends on the accuracy of the determination of the effective temperatures and gravities, as the instability strip ranges only around $1200 \mathrm{~K}$ in $T_{\text {eff }}$ and depends on gravity. With high SNR spectra for the bright sample, Bergeron et al. $(1995,2004)$ and Gianninas, Bergeron \& Fontaine $(2005,2006)$ find a pure instability strip, while there are $\sim 20$ stars inside the same instability strip if one uses the less accurate determinations of surface parameters for the fainter SDSS variables, and the relatively high detection limits of Mukadam et al. (2004) and Mullally et al. (2005). Castanheira et al. (2006c) find variability for two stars reported as nonvariables in the aforementioned searches, and Kepler et al. (2006) find the uncertainties in the SDSS parameters are a substantial fraction of the instability strip. Mukadam et al. (2006) suggest we can use the observed pulsation periods to determine $T_{\text {eff }}$, as there is a strong correlation between period and $T_{\text {eff }}$. Even with the small number of pulsations detected in the DAVs, seismology indicates hydrogen layer masses $M_{\mathrm{H}} \simeq 10^{-4}$ to $10^{-8} M_{*}$, an important limit in the study of chemical evolution of the surface composition of white dwarf stars due to diffusion, radiative levitation, and convection. The rotation periods derived from pulsation splittings are around $1 \mathrm{~d}$, consistent with those observed by line broadening. Velocity fields in line profiles start to be detected with time resolved spectra taken at the Keck $10 \mathrm{~m}$ telescopes. 


\section{Pulsations in DAs in Cataclysmic Variables}

Ten pulsators were discovered recently in low mass accretion systems (van Zyl et al. 2004; Nilsson et al. 2006), indicating the mass transfer does not strongly disturb the subsurface partial ionization zone that causes convection and/or pulsation. Accretion raises the external temperature distribution and changes external layers composition, but the underlying structure should be similar to single stars. The models have been calculated by Arras, Townsley \& Bildsten (2006).

Acknowledgments. We thank HELAS for partial support that made our participation in the conference possible.

\section{References}

Althaus L. G., Serenelli A. M., Panei J. A., et al., 2005, A\&A, 435, 631

Arras P., Townsley D. M., Bildsten L., 2006, ApJ, 643, L119

Barstow M. A., Holberg J. B., Grauer A. D., Winget D. E., 1986, ApJ, 306, L25

Beauchamp A., Wesemael F., Bergeron P., et al., 1999, ApJ, 516, 887

Bergeron P., Wesemael F., Lamontagne R., et al., 1995, ApJ, 449, 258

Bergeron P., Fontaine G., Billères M., Boudreault S., Green E. M., 2004, ApJ, 600, 404

Bond H. E., Grauer A. D., Green R. F., Liebert J. W., 1984, ApJ, 279, 751

Bradley P. A., Dziembowski W. A., 1996, ApJ, 462, 376

Brickhill A. J., 1991, MNRAS, 251, 673

Castanheira B. G., Kepler S. O., Moskalik P., et al., 2004, A\&A, 413, 623

Castanheira B. G., Kepler S. O., Mullally F., et al., 2006b, A\&A, 450, 227

Castanheira B. G., Kepler S. O., Costa A. F. M., et al., 2006c, A\&A, 462, 989

Castanheira B. G., Kepler S. O., Handler G., Koester D., 2006a, A\&A, 450, 331

Castanheira B. G., Nitta A., Kepler S. O., Winget D. E., Koester D., 2005, A\&A, 432, 175

Chanmugam G., 1972, Nat, 236, 83

Clemens J. C., van Kerkwijk M. H., Wu Y., 2000, MNRAS, 314, 220

Córsico A. H., Althaus L. G., 2005, A\&A, 439, L31

Córsico A. H., Althaus L. G., 2006, A\&A, 454, 863

Córsico A. H., Althaus L. G., Benvenuto O. G., Serenelli A. M., 2002, A\&A, 387, 531

Córsico A. H., Althaus L. G., Miller Bertolami M. M., 2006, A\&A, 458, 259

Córsico A. H., Althaus L. G., Montgomery M. H., García-Berro E., Isern J., 2005, A\&A, 429, 277

Córsico A. H., Benvenuto O. G., Althaus L. G., Isern J., García-Berro E., 2001, New Astron., 6, 197

Córsico A. H., García-Berro E., Althaus L. G., Isern J., 2004, A\&A, 427, 923

Costa J. E. S., Kepler S. O., Winget D. E., 1999, ApJ, 522, 973

Costa J. E. S., Kepler S. O., Winget D. E., et al., 2003, Baltic Astron., 12, 23

Dreizler S., 1998, Baltic Astron., 7, 71

Dolez N., Vauclair G., 1981, A\&A, 102, 375

Dolez N., Vauclair G., Kleinman S. J., et al., 2006, A\&A, 446, 237

Dziembowski W. A., 1977, Acta Astron., 27, 1

Dziembowski W. A., Koester D., 1981, A\&A, 97, 16

Eisenstein D. J., Liebert J., Harris H. C., et al., 2006, ApJS, 167, 40

Fujimoto M. Y., 1977, PASJ, 29, 331

Gianninas A., Bergeron P., Fontaine G., 2005, ApJ, 631, 1100 
Gianninas A., Bergeron P., Fontaine G., 2006, AJ, 132, 831

Goldreich P., Wu, Y., 1999, ApJ, 511, 904

Grauer A. D., Bond H. E., 1984, ApJ, 277, 211

Hansen B. M. S., Brewer J., Fahlman G. G., et al., 2002, ApJ, 574, L155

Iben I. Jr., 1982, ApJ, 260, 821

Ising J., Koester D., 2001, A\&A, 374, 116

Kanaan A., Nitta A., Winget D. E., et al., 2005, A\&A, 432, 219

Kawaler S., 1997, in Guinan E. F., Koch R. H., eds, 23rd meeting of the IAU, Stellar Evolution in Real Time. Astron. Soc. Pac., San Fracisco, p. 8

Kawaler S. D., Sekii T., Gough D. O., 1999, ApJ, 516, 349

Kawaler S. D., O'Brien M. S., Clemens J. C., et al., 1995, ApJ, 450, 350

Kawaler S. D., Potter E. M., Vučković M., et al., 2004, A\&A, 428, 969

Kawaler S. D., Winget D. E., Iben I. Jr., Hansen C. J., 1986, ApJ, 302, 530

Keeley D. A., 1979, in van Horn H. M., Weidemann V., eds, IAU Colloq. 53: White Dwarfs and Variable Degenerate Stars. University of Rochester, Rochester, p. 388

Kepler S. O., 1984, ApJ, 278, 754

Kepler S. O., Castanheira B. G., Costa A. F. M., Koester D., 2006, MNRAS, 372, 1799

Kepler S. O., Nather R. E., Winget D. E., et al., 2003, A\&A, 401, 639

Kepler S. O., Castanheira B. G., Saraiva M. F. O., et al., 2005a, A\&A, 442, 629

Kepler S. O., Costa J. E. S., Castanheira B. G., et al., 2005b, ApJ, 634, 1311

Kepler S. O., Nather R. E., McGraw J. T., Robinson E. L., 1982, ApJ, 254, 676

Kepler S. O., Robinson E. L., Koester D., et al., 2000, ApJ, 539, 379

Kepler S. O., Robinson E. L., Nather R. E., 1983, ApJ, 271, 744

Kleinman S. J., Nather R. E., Winget D. E., et al., 1998, ApJ, 495, 424

Kotak R., van Kerkwijk M. H., Clemens J. C., 2002, A\&A, 388, 219

Kotak R., van Kerkwijk M. H., Clemens J. C., 2004, A\&A, 413, 301

Kotak R., van Kerkwijk M. H., Clemens J. C., Bida T.A., 2002, A\&A, 391, 1005

Kotak R., van Kerkwijk M. H., Clemens J. C., Koester D., 2003, A\&A, 397, 1043

Landolt A. U., 1968, ApJ, 153, 151

Lasker B. M., Hesser J. E., 1969, ApJ, 158, L171

Lasker B. M., Hesser J. E., 1971, ApJ, 163, L89

Metcalfe T. S., 2003, ApJ, 587, L43

Metcalfe T. S., Montgomery M. H., Kawaler S. D., 2003, MNRAS, 344, L88

Metcalfe T. S., Salaris M., Winget D. E., 2002, ApJ, 573, 803

McGraw J. T., 1979, ApJ, 229, 203

McGraw J. T., Robinson E. L., 1975, ApJ, 200, L89

McGraw J. T., Robinson E. L., 1976, ApJ, 205, L155

McGraw J. T., Starrfield S. G., Angel J. R. P., Carleton N. P., 1979, SAO Special Report, 385, 125

Montgomery M. H., 2005, ApJ, 633, 1142

Montgomery M. H., 2006, Mem. Soc. Astron. Ital., 77, 464

Mukadam A. S., Kepler S. O., Winget D. E., et al., 2003, ApJ, 594, 961

Mukadam A. S., Mullally F., Nather R. E., et al., 2004, ApJ, 607, 982

Mukadam A. S., Montgomery M. H., Winget D. E., Kepler S. O., Clemens J. C., 2006, ApJ, 640, 956

Mullally F., Thompson S. E., Castanheira B. G., et al., 2005, ApJ, 625, 966

Nilsson R., Uthas H., Ytre-Eide M., Solheim J.-E., Warner B., 2006, MNRAS, 370, L56 
Nitta A., Kleinman S. J., Krzesinski J., et al., 2005, in Koester D., Moehler S., eds, ASP Conf. Ser. Vol. 334, 14th European Workshop on White Dwarfs. Astron. Soc. Pac., San Francisco,p. 585

O'Brien M. S., Vauclair G., Kawaler S. D., et al., 1998, ApJ, 495, 458

Quirion P.-O., Fontaine G., Brassard P., 2004, ApJ, 610, 436

Quirion P.-O., Fontaine G., Brassard P., 2005, A\&A, 441, 231

Quirion P.-O., Fontaine G., Brassard P., 2006, Mem. Soc. Astron. Ital., 77, 53

Quirion P.-O., Fontaine G., Brassard P., 2007, 2007, in Napiwotzki R., Burleigh M., eds, 15th European Workshop on White Dwarfs, ASP Conf. Ser., in press (astro-ph/0610443)

Robinson E. L., Kepler S. O., Nather R. E., 1982, ApJ, 259, 219

Robinson E. L., Nather R. E., McGraw J. T., 1976, ApJ, 210, 211

Robinson E. L., Stover R. J., Nather R. E., McGraw J. T., 1978, ApJ, 220, 614

Schoenberner D., 1979, A\&A, 79, 108

Starrfield S. G., Cox A. N., Hodson S. W., Pesnell W. D., 1983, ApJ, 268, L27

Stover R. J., Nather R. E., Robinson E. L., Hesser J. E., Lasker B. M., 1980, ApJ, 240, 865

Thompson S. E., Clemens J. C., van Kerkwijk M. H., Koester D., 2003, ApJ, 589, 921

van Zyl L., Warner B., O'Donoghue D., et al., 2004, MNRAS, 350, 307

Vauclair G., Solheim J.-E., Østensen R. H., 2005, A\&A, 433, 1097

Voss B., Koester D., Østensen R., et al., 2006, A\&A, 450, 1061

Vuille F., 2000, MNRAS, 313, 170

Vuille F., O'Donoghue D., Buckley D. A. H., et al., 2000, MNRAS, 314, 689

Yeates C. M., Clemens J. C., Thompson S. E., Mullally F., 2005, ApJ, 635, 1239

Warner B., Robinson E. L., 1972, Nat, 239, 2

Winget D. E., Nather R. E., Clemens J. C., et al., 1994, ApJ, 430, 839

Winget D. E., Cochran W. D., Endl M., et al., 2003, in Deming D., Seager S., eds, ASP Conf. Ser. Vol. 294, Scientific Frontiers in Research on Extrasolar Planets. Astron. Soc. Pac., San Francisco, p. 59

Winget D. E., Hansen C. J., Liebert J., et al., 1987, ApJ, 315, L77

Winget D. E., Hansen C. J., van Horn H. M., 1983, Nat, 303, 781

Winget D. E., Kepler S. O., Kanaan A., Montgomery M. H., Giovannini O., 1997, ApJ, 487, L191

Winget D. E., van Horn H. M., Hansen C. J., 1981, ApJ, 245, L33

Winget D. E., Robinson E. L., Nather R. E., Fontaine G., 1982a, ApJ, 262, L11

Winget D. E., van Horn H. M., Tassoul M., et al., 1982b, ApJ, 252, L65

Winget D. E., Robinson E. L., Nather R. E., Kepler S. O., O'Donoghue D., 1985, ApJ, 292, 606

Winget D. E., Sullivan D. J., Metcalfe T. S., Kawaler S. D., Montgomery M. H., 2004, ApJ, 602, L109

Wu Y., 2001, MNRAS, 323, 248

\section{DISCUSSION}

Hatzes: I was really amazed how dramatic the power spectrum of GD 358 changed. Do you worry that you are missing detail because of poor temporal sampling?

Kepler: Since the modes came back at the same frequencies after the dramatic power change, we believe that there are no time scales shorter than a month involved. Since we need about 20 telescopes looking at the same star we can only do such a project every three or four years.

Bedding: You need very large telescopes to measure mmag changes in a 22nd magnitude star. How much time do you need to do useful science? You can get, say, two or three nights on such telescopes, but not weeks.

Kepler: It depends on what you want to do. If you really want to do seismology, you need lots of nights, but we can detect the pulsations in a couple of hours. You would need a couple of nights over two or three seasons to do seismology. 